\title{
Right Ventricular Systolic and Diastolic Function at Rest in Patients with Coronary Artery Disease
}

\section{Assessment with Equilibrium Radionuclide Ventriculography*}

\author{
Nail M. Caglar, M.D., Haruo Araki, M.D., Yuji Taira, M.D., \\ Takaya Fukuyama, M.D., and Motoomi Nakamura, M.D.
}

\section{SummaRY}

Right ventricular systolic and diastolic function was studied in patients with ischemic heart disease using equilibrium radionuclide ventriculography. In patients with inferior myocardial infarction and proximal right coronary lesions, the right ventricular ejection fraction $(0.43 \pm 0.06, n=10$, mean $\pm S D)$ and peak filling rate $(1.7 \pm 0.4 \mathrm{EDV} / \mathrm{sec})$ were lower than normals $(0.57 \pm 0.07$ and 2.7 $\pm 0.4 \mathrm{EDV} / \mathrm{sec}, \mathrm{n}=10, \mathrm{p}<0.001$, respectively). In these patients, the right ventricular time to peak filling rate was longer than in normals $(225 \pm 36 \mathrm{msec}$ vs $136 \pm 45 \mathrm{msec}, \mathrm{p}<0.001)$, while the left ventricular ejection fraction remained normal. In patients with inferior myocardial infarction and distal right coronary lesions, the right ventricular ejection fraction, peak filling rate and time to peak filling rate were not different from those in normals. Even in patients with proximal right coronary lesions, the right ventricular ejection fraction was normal unless they had an inferior myocardial infarction. A decreased left ventricular ejection fraction and abnormal motion of the ventricular septum did not affect the right ventricular ejection fraction. The present results suggest that patients with an inferior myocardial infarction and proximal right coronary lesion often develop right ventricular systolic and diastolic dysfunction.

\section{Additional Indexing Words :}

Right ventricular function Coronary artery disease Inferior myocardial infarction Radionuclide ventriculography

From the Research Institute of Angiocardiology and Cardiovascular Clinic, Faculty of Medicine, Kyushu University, Fukuoka, Japan.

* The study was supported in part by Takeda Medical Research Foundation.

Address for reprint: Motoomi Nakamura, M.D., Research Institute of Angiocardiology and Cardiovascular Clinic, Faculty of Medicine, Kyushu University, 3-1-1 Maidashi, Higashi-ku, Fukuoka 812, Japan.

Received for publication May 28, 1984.

Manuscript revised August 29, 1984. 
$7 \mathrm{HE}$ impairment of right ventricular function after left ventricular myo-

1 cardial infarction suggests that the area of myocardial infarction involves the right ventricular free wall and these cases are associated with stenosis of the right coronary artery. ${ }^{1), 2)}$ Although contrast angiographic methods have been developed to determine the right ventricular ejection fraction, ${ }^{3)-5)}$ equilibrium radionuclide ventriculography is a simple and noninvasive approach for the determination of the right ventricular ejection fraction, independent the geometry of the right ventricle. ${ }^{6)-8}$ There are few studies investigating the effects of significant stenosis of right coronary artery on the resting right ventricular ejection fraction in ischemic heart disease. Diastolic performance of the left ventricle was recently investigated with radionuclide techniques. But, to our knowledge, there is no previously published study on right ventricular diastolic function in normals and in patients with ischemic heart disease using equilibrium radionuclide ventriculography. This study used equilibrium radionuclide ventriculography to evaluate the effect of the location of right coronary artery stenosis in patients with ischemic heart disease on right ventricular systolic and diastolic functions at rest.

\section{Materials and Methods}

\section{Study population}

Multiple gated equilibrium radionuclide ventriculography was performed on 35 patients and 10 normal subjects. No patient had either valvular heart disease or clinical or radiographic evidence of pulmonary disease. Pulmonary function tests were obtained routinely and were normal in each case. Patients with bundle branch block or arrhythmia were not included in the radionuclide study. All patients were clinically class I or class II according to the New York Heart Association classification. Infarct location was defined on the surface electrocardiogram using standard criteria as inferior (including inferolateral and inferoposterior) or anterior (including anteroseptal and anterolateral). Forty-one patients underwent left and right heart catheterization and selective coronary angiography. Hemodynamic studies were performed within 30-40 days following acute myocardial infarction. The interval between radionuclide ventriculography and the hemodynamic study was 1 to 2 weeks. The motion of the interventricular septum was analyzed in the left anterior oblique projection using contrast X-ray ventriculography. The coronary arteriograms were interpreted by a cardiologist without knowledge of the results of radionuclide studies. A significant obstructive lesion was defined by the presence of at least a $75 \%$ decrease in luminal diameter when seen in two projections. Stenoses of the right coronary artery were defined 
as either proximal or distal. The site of stenosis was considered proximal if there was a significant stenosis of the right coronary artery before the acute marginal branch.

The study population was divided intio 5 groups depending on the coronary artery lesions and the type of infarction.

Group 1: Ten normal subjects were examined. Four were normal volunteers without abnormal cardiopulmonary findings. The remaining 6 subjects were patients who underwent diagnostic cardiac catheterization for chest pain. All had normal coronary arteriograms and hemodynamic results. All but one were male. The mean of age was $33 \pm 12$ years (mean $\pm S D$, range: 20 to 55 years).

Group 2: This group consisted of 10 patients. All but one were male, mean age $54 \pm 11$ (range: 31 to 72 years). All patients had significant stenosis of the proximal right coronary artery with an inferior myocardial infarction and no other type of infarction. There were no significant lesions in the distal right coronary artery, neither in the left circumflex nor the left anterior descending coronary artery. The stenosis was proximal to the right ventricular branch in 9 patients and distal to the right ventricular branch but proximal to the acute marginal branch in the remaining patient. Six patients had total occlusion, 1 had subtotal occlusion and the remaining 3 patients had $90 \%$ stenosis.

Group 3: This group consisted of 7 male patients. Their mean age was $55 \pm 6$ years (range: 47 to 62 years). All had significant stenosis limited in the distal right coronary artery or left circumflex with only an inferior myocardial infarction. There was neither a significant proximal right coronary artery stenosis nor an anterior myocardial infarction.

Group 4: Twelve patients had anterior myocardial infarctions with significant lesions of the left coronary artery. None of the them had significant stenosis of the right coronary artery and inferior myocardial infarction. Eleven of them were male and 1 female. Their mean age was $52 \pm 10$ years (range: 24 to 62 years).

Group 5: This group consisted of 6 male patients. Their mean age was $54 \pm 4$ years (range: 39 to 65 years). All had significant proximal right coronary stenosis without history or electrocardiographic evidence of infarction. The stenosis was proximal to the right ventricular branch in all patients. Three patients had total occlusion, 1 had subtotal stenosis and 2 patients had $75 \%$ stenosis. All had well developed collaterals to right coronary artery.

Multiple gated blood pool imaging was performed following the injec- 
tion of $15 \mathrm{mCi}{ }^{99 \mathrm{~m}} \mathrm{Tc}$-labeled human serum albumin using a gamma camera (PHO/Gamma LFOV Searle Radiographic Inc.) and a converging hole collimator (Searle). The camera was positioned in the $45^{\circ}$ left anterior oblique projection mode with caudal tilt. The energy selection was set at $140 \mathrm{KeV}$ with a $20 \%$ window. Gated images were collected with a computer and an electrocardiographic synchronizer in a $64 \times 64$ matrix using a commercially available program for nuclear cardiology (Shimadzu, Scintipac 1200). The frame rate for each patient was at least $40 \mathrm{msec} / \mathrm{frame} .{ }^{9}{ }^{9}$ Smoothing was performed using a spatial ( 9 point) and a temporal ( 3 point) filter. Background correction was estimated automatically by the computer using a linear interpolation technique similar to that described by Goris et al. ${ }^{10)}$

A right ventricular region of interest on the diastolic image of composite cardiac cycle was drawn with a joy-stick to a width of 2-3 matrix elements from the right ventricular cavity with care taken to avoid the inclusion of the pulmonary artery. An automated computer program then defined the right ventricular regions of interest for each frame of the composite cardiac cycle by a threshold method, determined the activity within each region of interest and generated a time-activity curve based on the background corrected counts obtained from these varying regions of interest. The sampled region of interest on each frame was displayed on CRT and its accuracy was examined by an operator. The computer program permitted the operator to observe the motion of each cardiac chamber on a color CRT and helped to determine the end-diastolic region of interest and to examine the accuracy of the automatically drawn region of interest for each frame. The right ventricular ejection fraction was determined by the computer from the time activity curve. A first derivative was calculated from the time activity curve.

The peak diastolic filling rate and time to peak filling rate were determined from the first derivative curve by the computer. The late positive peak of the first derivative curve was defined as peak filling rate. This value was divided by end-diastolic maximum counts to normalize the measurements and expressed as end-diastolic counts per second. ${ }^{11)}$ The time to peak filling rate was defined as the time between the nadir of the time activity curve and the late peak of the first derivative curve. ${ }^{11}$ The left ventricular ejection fraction was determined by the same method.

To determine the interobserver and intraobserver variability for the right and left ventricular ejection fraction, the right ventricular peak filling rate and the time to peak filling rate, 12 cases were randomly selected and analyzed. The right ventricular ejection fraction measurement obtained by multiple gated equilibrium radionuclide ventriculography were compared with 
first pass in 24 cases.

First pass radionuclide ventriculography was performed in the $30^{\circ}$ right anterior oblique projection after a bolus injection of $15 \mathrm{mCi} 99 \mathrm{~m}$ Tc-labeled human serum albumin, using a gamma camera with a nuclear medicine computer (Shimadzu, Scintipac 1200). Data were collected in the list mode. From the low frequency time activity curve, the right ventricular activity of 4 beats was selected and multiple gated imaging was made using the same method applied to the equilibrium study. A frame interval of $40 \mathrm{msec}$ was used.

\section{Statistical analysis}

The values were compared by analysis of variance and considered to be statistically significant at a $\mathrm{p}$ value less than 0.05 . All values were expressed as mean $\pm \mathrm{SD}$.

\section{Results}

Right and left ventricular ejection fractions

The right and left ventricular ejection fractions of the 5 groups are shown in Fig. 1. In normal subjects, the right ventricular ejection fraction ranged from 0.46 to 0.67 (mean $0.57 \pm 0.07, \mathrm{n}=10$ ) and the left ventricular ejection fraction from 0.56 to 0.78 (mean $0.70 \pm 0.06$ ). There was no significant difference in the right ventricular ejection fraction between the normal subjects and Groups 3, 4 and 5. In the group with stenosis of the proximal right coronary artery and an inferior myocardial infarction (Group 2), the mean right ventricular ejection fraction was $0.43 \pm 0.06$ and was significantly lower than those in other 4 groups $(\mathrm{p}<0.001$ for all groups). The left ventricular ejection fraction in Group 4, which consisted of patients with an anterior myocardial infarction, was significantly lower than others, while the right ventricular ejection fraction of the group was normal.

\section{Right ventricular diastolic indices}

Fig. 2 shows 2 parameters of right ventricular diastolic function in normal subjects and 4 groups of patients. As shown in the upper panel, the right ventricular peak filling rate was lower in patients with proximal right coronary artery stenosis $(1.7 \pm 0.4 \mathrm{EDV} / \mathrm{sec}$ in Group 2 and $1.9 \pm 0.6$ in Group 5) than in normals $(2.7 \pm 0.4 \mathrm{EDV} / \mathrm{sec}, \mathrm{p}<0.001)$. The right ventricular time to peak filling rate was significantly longer in Group 2 (225 $\pm 36 \mathrm{msec})$ compared to that in normals $(136 \pm 45 \mathrm{msec}, \mathrm{p}<0.001)$, while heart rate was not different among these 5 groups of patients. 

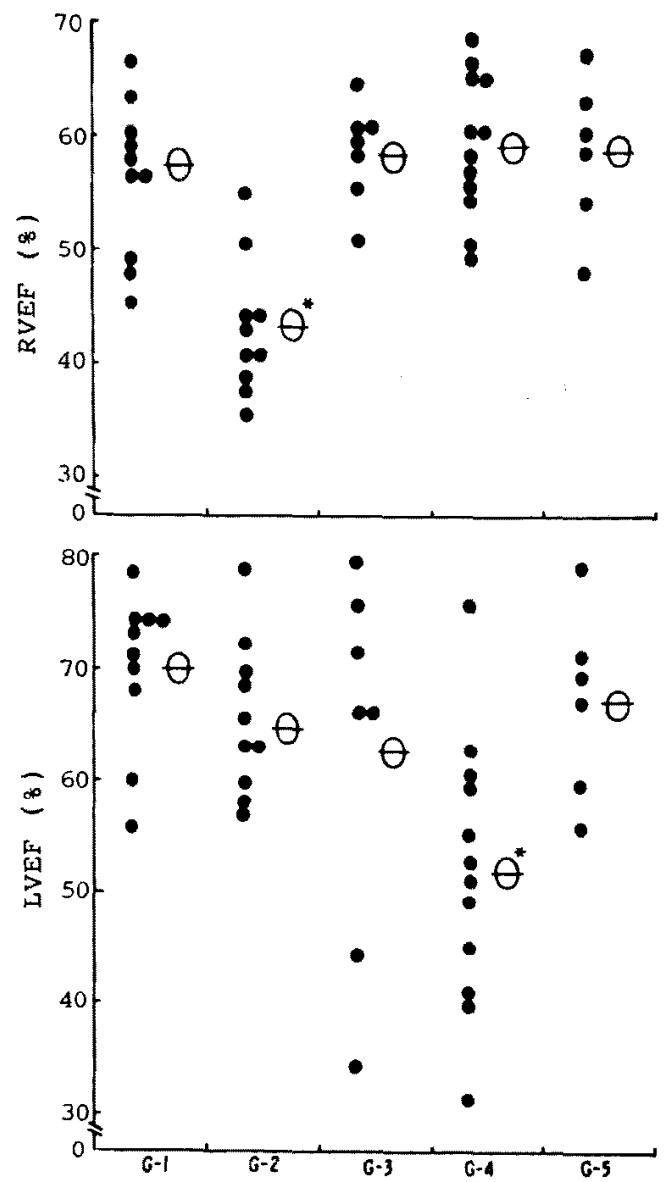

Fig. 1. Right and left ventricular ejection fraction values in normal subjects (G-1), patients with proximal right coronary artery stenosis and inferior myocardial infarction (G-2), patients with distal right coronary artery stenosis and inferior myocardial infarction (G-3), patients with only significant left coronary artery stenosis and anterior myocardial infarction (G-4) and patients with proximal right coronary artery stenosis without myocardial infarction (G-5). Open circles with horizontal bars indicate mean values. The mean right ventricular ejection fraction (RVEF) in G-2 was lower than in normal subjects $(\mathrm{p}<0.001)$ and the mean left ventricular ejection fraction (LVEF) in G-4 was lower than in normal subjects $(\mathrm{p}<$ $0.001)$.

Effect of interventricular septal motion on right ventricular ejection fraction

Fig. 3 summarizes the relationship between the right ventricular ejection fraction and abnormalities of the interventricular septum, determined by Xray contrast ventriculography. Patients with an akinetic or a dyskinetic interventricular septum did not necessarily have a decreased right ventricular ejection fraction and some patients with normal motion of the interventricular 

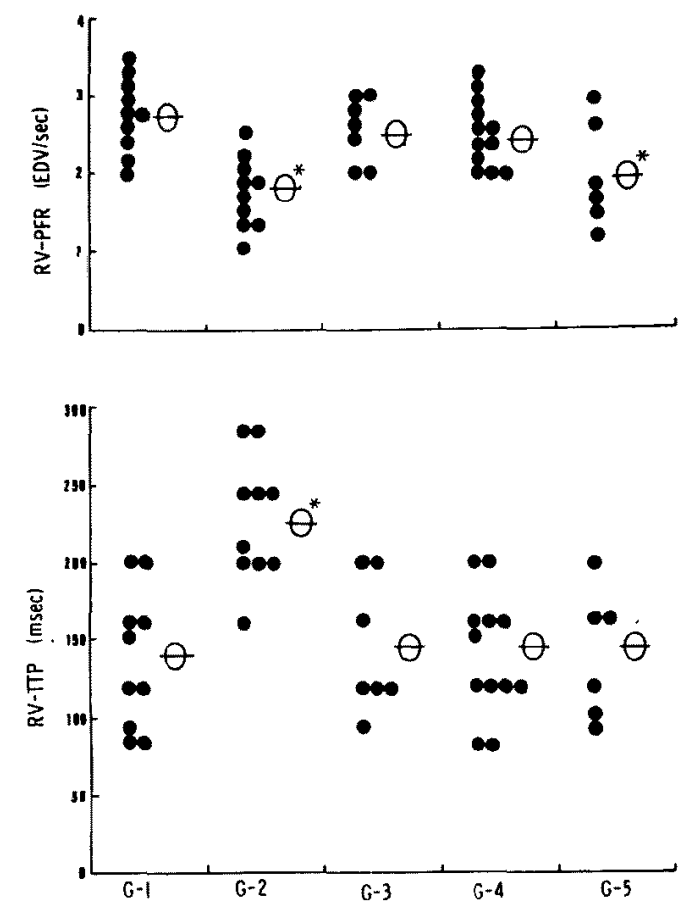

Fig. 2. Right ventricular pcak filling ratc (RV-PFR) and time to peak filling rate (RV-TTP) values of Group 1 (G-1, normal subjects), Group 2 $(G-2$, proximal right coronary artery stenosis with inferior myocardial infarction), Group 3 (G-3, distal right coronary artery stenosis with inferior myocardial infarction), Group 4 (G-4, left coronary artery stenosis with anterior myocardial infarction) and Group 5 (G-5, proximal right coronary artery stenosis without myocardial infarction). Open circles with horizontal. bars indicate mean values.

* $\mathrm{p}<0.001$ versus normals.

septum had a decreased right ventricular ejection fraction. This indicates that the right ventricular ejection fraction is independent of the motion of the interventricular septum.

\section{Validation of data}

To evaluate the reproducibility of radionuclide results, inter- and intraobserver variabilities were analyzed in 12 patients. These variations are expressed by the correlation coefficients ( $r$ ), the equations of regression lines and the standard error of the estimates. As shown in Table I, there was a satisfactory reproducibility in all 4 parameters of right ventricular ejection fraction, peak filling rate and time to peak filling rate, and left ventricular ejection fraction. There was a good correlation between right ventricular ejection fractions calculated by first pass and the equilibrium method with 


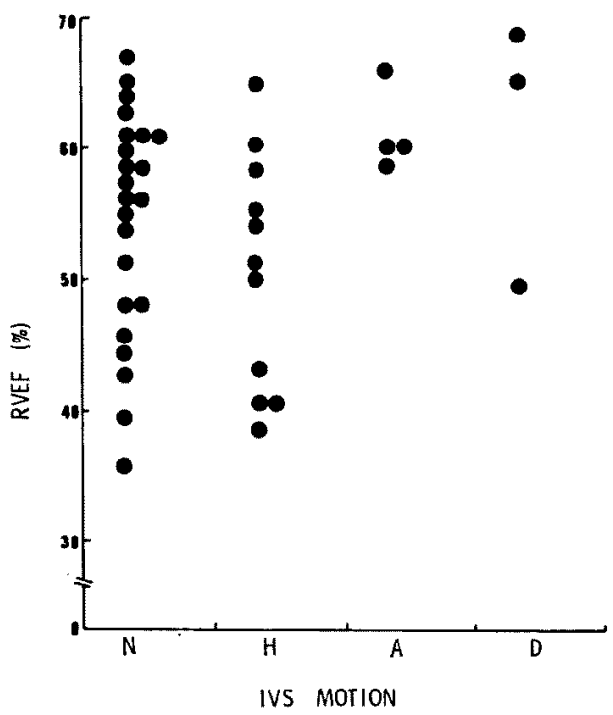

Fig. 3. The relationship between right ventricular ejection fraction (RVEF) and abnormalities of interventricular septum (IVS) in coronary patients. $\mathrm{N}=$ normal; $\mathrm{H}=$ hypokinetic; $\mathrm{A}=$ akinetic; $\mathrm{D}=$ dyskinetic.

Table I. Inter- and Intraobserver Variabilities $(n=12)$

\begin{tabular}{|c|c|c|c|c|c|c|}
\hline & \multicolumn{3}{|c|}{ Interobserver Variabilities } & \multicolumn{3}{|c|}{ Intraobserver Variabilities } \\
\hline & $\begin{array}{l}\text { Corre- } \\
\text { lation } \\
\text { coefficient }\end{array}$ & $\begin{array}{l}\text { Regression } \\
\text { Equation }\end{array}$ & $\begin{array}{l}\text { Standard } \\
\text { error of } \\
\text { estimate }\end{array}$ & $\begin{array}{l}\text { Corre- } \\
\text { lation } \\
\text { coefficient }\end{array}$ & $\begin{array}{l}\text { Regression } \\
\text { Equation }\end{array}$ & $\begin{array}{l}\text { Standard } \\
\text { error of } \\
\text { estimate }\end{array}$ \\
\hline $\begin{array}{l}\text { Right ventricular } \\
\text { ejection fraction }\end{array}$ & 0.90 & $y=0.04+0.92 x$ & 0.04 & 0.96 & $y=0.02+0.98 x$ & 0.03 \\
\hline $\begin{array}{l}\text { Right ventricular } \\
\text { peak filling rate }\end{array}$ & 0.88 & $y=0.88+0.86 x$ & 0.39 & 0.91 & $y=0.57+0.91 x$ & 0.36 \\
\hline $\begin{array}{l}\text { Right ventricular } \\
\text { time to peak filling } \\
\text { rate }\end{array}$ & 0.84 & $y=11.7+0.85 x$ & 24 & 0.90 & $y=6.3+0.91 x$ & 17 \\
\hline $\begin{array}{l}\text { Left ventricular } \\
\text { ejection fraction }\end{array}$ & 0.93 & $y=0.05+0.91 x$ & 0.02 & 0.94 & $y=0.05+0.92 x$ & 0.02 \\
\hline
\end{tabular}

a variable region of interest $(\mathrm{r}=0.92, \mathrm{y}=0.029+0.96 \mathrm{x}, \mathrm{n}=24)$.

\section{Discussion}

Equilibrium gated radionuclide ventriculography using a variable right ventricular region of interest has been demonstrated to correlate well with first pass methods on measuring the right ventricular ejection fraction. ${ }^{6), 12)}$ Our right ventricular ejection fraction values in normal subjects were compatible with the reported results of both first pass ${ }^{8,13), 14}$ and equilibri- 
um radionuclide ventriculography with a variable region of interest. ${ }^{15), 16)}$ Furthermore, good correlation between the right ventricular ejection fractions calculated by first pass and equilibrium methods was obtained in our study $(\mathrm{r}=0.92, \mathrm{n}=24)$. Thus, the value of right ventricular ejection fraction in the present study seems to be reliable.

Effect of right coronary artery stenosis on the right ventricular ejection fraction

The present study showed that the right ventricular ejection fraction of Group 2 was significantly lower than normal. This result seems very reasonable, since Group 2 consisted of patients with an inferior myocardial infarction and proximal right coronary stenosis. After myocardial infarction, the right ventricular free wall will also become ischemic or even necrotic in these patients. Actually, some of these patients showed a rise in right ventricular diastolic pressure and right atrial pressure, with a normal pulmonary arterial wedge pressure in their acute phase of myocardial infarction (a sign of right ventricular infarction). However, they did not show signs of congestive right ventricular failure at the time of this study.

The right ventricular ejection fraction in Group 3 were within the normal range. Group 3 consisted of patients with an inferior myocardial infarction and with right coronary lesions distal to the acute marginal branches. Thus, right ventricular function should remain normal, despite inferior myocardial infarctions in these patients.

Group 5 consisted of patients with proximal right coronary lesions but without myocardial infarction. The right ventricular ejection fractions in these patients were within normal limits. In 3 of these patients, total occlusion of the proximal right coronary artery was found and its distal area, including the right ventricle, was perfused through well developed collaterals. This indicates that the right ventricular systolic function at rest may be preserved by the blood supply through collaterals alone. Similarly, Berger et al did not find any relationship between the presence of proximal right coronary lesions and abnormal right ventricular ejection fraction at rest using the first pass technique; only one of their patients had an inferior myocardial infarction. ${ }^{14}$ )

In the present study, patients with restricted, proximal right coronary artery stenosis and an inferior myocardial infarction (Group 2) had low right ventricular ejection fraction. Previous observations have shown that depressed right ventricular function in ischemic heart disease occurs when an inferior myocardial infarction extends into the free wall of the right ventricle and that isolated right ventricular damage is rare.11,2),17) Thus, a low right ventricular ejection fraction in Group 2 may indicate that the free wall of the 
right ventricle is also involved in addition to the left ventricular inferior infarction.

Effect of anterior myocardial infarctions on the right ventricular ejection fraction

Patients in Group 4 had an anterior myocardial infarction with left coronary lesions. They did not have proximal right coronary lesions or inferior myocardial infarction. They had anteroseptal, anterolateral or extensive anterior infarction, and the left ventricular ejection fraction was significantly lower than normal. The right ventricular ejection fraction, however, remained normal. This may indicate that the right ventricular systolic function does not depend on the left ventricular systolic function. Some of the patients in this group showed abnormal interventricular septal motion on contrast $\mathrm{X}$-ray ventriculography (akinesis in 4 and dyskinesis in 3 ). The right ventricular ejection fractions were normal in these patients, indicating that abnormal septal motion may not influence right ventricular systolic function.

Effects of right coronary artery stenosis on right ventricular diastolic function

There are no previously published radionuclide ventriculography studies concerning the right ventricular peak filling rate and time to peak filling rate in coronary patients. In the present study, the right ventricular peak filling rate was significantly lower in Group 2 than that in normals. The peak filling rate and time to peak filling rate in Groups 3 and 4 were not statistically different from normals. The right ventricular peak filling rate decreased in $67 \%$ of Group 5 patients who had significant stenosis of the proximal right coronary artery without infarction and a normal right ventricular ejection fraction. The mechanism of this abnormal peak filling rate in patients with a normal ejection fraction is not clear. During early diastole, myocardial relaxation is an active, energy-dependent process. Previous investigations have shown that hypoxia may impair the rate of myocardial relaxation. ${ }^{18)}$ Mild ischemia of the right ventricle may produce changes in filling without altering the systolic function.

In the absence of quantitative right ventricular volume estimation, the right ventricular peak filling rate cannot be expressed as absolute volume changes. Radionuclide ventriculography provides a gross approximation of diastolic function because only relative volumes are determined and expressed as a function of end-diastolic counts. The mechanism responsible for prolongation of the time to peak filling rate cannot specifically be delineated without knowing the timing of valvular opening and closing. This cannot be provided by analysis of the radionuclide time activity curve alone. Nevertheless, radionuclide ventriculography and the computer algorithm used to 
derive these diastolic indices are reproducible, given the very low observed variability of these measurements. Despite these methodological limitations, our data suggest that abnormalities in right ventricular diastolic filling can be detected using equilibrium radionuclide ventriculography and that these abnormalities are independent of right ventricular systolic function in some patient populations. Abnormalities of diastolic filling are seen in almost all patients with previous inferior myocardial infarctions and significant stenosis of the proximal right coronary artery. These abnormalities are also common in coronary patients without previous left ventricular infarction but with significant stenosis of the proximal right coronary artery and normal right ventricular systolic function. Such diastolic abnormalities may be the earliest manifestation of ischemic right ventricular dysfunction.

\section{ACKNOWLEDGMENTS}

The authors thank Seigi Irie, Masao Sakuragi, Yuichi Fukano and Masakimi Abo for technical assistance, Toshio Inoue for statistical assistance and Tomoko Hirokawa for secretarial assistance.

\section{REFERENCES}

1. Isner J, Roberts W: Right ventricular infarction complicating left ventricular infarction secondary to coronary heart disease: frequency, location, associated findings and significance from analysis of 236 necropsy patients with acute or healed myocardial infarction. Am J Cardiol 42: 885,1978

2. Cohn J, Guiha N, Broder M, Limas C: Right ventricular infarction: clinical and hemodynamic features. Am J Cardiol 35: 209, 1974

3. Boak JG, Kreulen T, Spann T: A geometric basis for the calculation of right ventricular volume in man. Cath Cardiovasc Diagn 3: 217, 1977

4. Arcilla $R$, Tias $P$, Thilenius $O$, Raninger $K$ : Angiographic method for volume estimation of right and left ventricles. Chest 60:446, 1971

5. Slutsky $R$, Bhargawa V, Dittrich $H$, Castello D: Comparison of single plane and biplane contrast analyses of right ventricular function and size. Am Heart J 104: 100, 1982

6. Maddahi J, Berman D, Matsuoka D, Waxman AD, Stankus KE, Forrester JS: A New technique for assessing right ventricular ejection fraction using rapid multiple gated equilibrium cardiac blood pool scintigraphy. Description, validation and findings in chronic coronary artery disease. Circulation 60: 581,1979

7. Maddahi J, Berman D, Matsuoka D, Waxman A, Forrester J, Swan H: Right ventricular ejection fraction during exercise in normal subjects and in coronary artery disease patients: assessment by multiple gated equilibrium scintigraphy. Circulation 62: 133, 1980

8. Slutsky R, Hooper W, Gerber K, Battler A, Froelicher V, Ashburn W, Karliner J : Assessment of right ventricular function at rest and during exercise in patients with coronary heart disease. A new approach using equilibrium radionuclide angiography. Am J Cardiol 45: 63, 1980

9. Bacharach SL, Green MV, Borer JS, Hyde JE, Farkas SP, Johnson GS: Left ventricular peak ejection rate, filling rate and ejection fraction-frame rate requirements at rest and exercise: concise communication. J Nucl Med 20: 189, 1979

10. Goris ML, Daspit SG, McLaughlin P, Kriss JP: Interpolative background substraction. J Nucl Med 17: 744, 1976 
11. Mancini JGB, Slutsky RA, Norris SL, Bhargava V, Ashburn WL, Higgins CB: Radionuclide analysis of peak filling rate, filling fraction and time to peak filling rate. Response to supine bicycle exercise in normal subjects and patients with coronary disease. Am J Ciardiol 51 : 43,1983

12. Holman BL, Wynne J, Zielonka JS, Idoine JD: A simplified technique for measuring right ventricular ejection fraction using the equilibrium radionuclide angiogram and the slant-hole collimator. Radiology 138: 429, 1981

13. Tobinick E, Schelbert HR, Henning H: Right ventricular ejection fraction in patients with acute anterior and inferior myocardial infarction. Assessed by radionuclide angiography. Circulation 57: 1078, 1978

14. Berger H, Johnstone DE, Sands JM, Gottschalk A, Zaret BL: Response of right ventricular ejection fraction to upright bicycle exercise in coronary artery disease. Circulation 60: 1292 , 1979

15. Manyari DE, Kostuk WJ: Left and right ventricular function at rest and during bicycle exercise in the supine and sitting positions in normal subjects and patients with coronary artery disease. Assessment by radionuclide ventriculography. Am J Cardiol 51: 36, 1983

16. Legrand V, Chevique M, Foulon J, Rigo P: Evaluation of right ventricular function by gated blood-pool scintigraphy. J Nucl Med 24: 886, 1983

17. Rigo P, Murray M, Taylor DR, Weisfeldt ML, Kelly DT, Strauss HW, Pitt B: Right ventricular dysfunction detected by gated scintigraphy in patients with acute inferior myocardial infarction. Circulation 52: 268, 1975

18. Weisfeldt ML, Armstrong P, Sully HE, Sanders CA, Dagget WM: Incomplete relaxation between beats after myocardial hypoxia and ischemia. J Clin Invest 53: 1626, 1974 